\title{
AUTOMATIC RECONSTRUCTION OF ROOF MODELS FROM BUILDING OUTLINES AND AERIAL IMAGE DATA
}

\author{
Vojtěch Hron*, Lena Halounová \\ Czech Technical University in Prague, Faculty of Civil Engineering, Department of Geomatics, Thákurova 7, \\ 16629 Prague, Czech Republic \\ * corresponding author: vojtech.hron@fsv.cvut.cz
}

\begin{abstract}
The knowledge of roof shapes is essential for the creation of 3D building models. Many experts and researchers use 3D building models for specialized tasks, such as creating noise maps, estimating the solar potential of roof structures, and planning new wireless infrastructures. Our aim is to introduce a technique for automating the creation of topologically correct roof building models using outlines and aerial image data. In this study, we used building footprints and vertical aerial survey photographs. Aerial survey photographs enabled us to produce an orthophoto and a digital surface model of the analysed area. The developed technique made it possible to detect roof edges from the orthophoto and to categorize the edges using spatial relationships and height information derived from the digital surface model. This method allows buildings with complicated shapes to be decomposed into simple parts that can be processed separately. In our study, a roof type and model were determined for each building part and tested with multiple datasets with different levels of quality. Excellent results were achieved for simple and medium complex roofs. Results for very complex roofs were unsatisfactory. For such structures, we propose using multitemporal images because these can lead to significant improvements and a better roof edge detection. The method used in this study was shared with the Czech national mapping agency and could be used for the creation of new 3D modelling products in the near future.
\end{abstract}

KEYWORDS: Building reconstruction, roof model, edge detection, orthophoto, digital surface model, GIS.

\section{INTRODUCTION}

Experts from a wide range of disciplines use complex spatial data to solve specialized tasks, such as creating noise maps, highway inventory [1], modelling air pollution, estimating the solar potential of roof structures, planning new wireless infrastructures, designing houses by taking natural daylight requirements into consideration and generating virtual environments for flight simulators. These tasks require the use of digital elevation models and 3D building models with generalized roof structures also referred to as Level of Detail 2 (LoD2) buildings [2]. The wide interest in LoD2 building models has led to their possible inclusion in the INSPIRE Buildings theme [3. Currently, digital elevation models, such as the digital terrain model (DTM) and the digital surface model (DSM), are typically already available on the market and are widely used. 3D LoD2 building models (subsequently referred to as "3D building models") are available only for certain areas, mostly urban areas, in the form of $3 \mathrm{D}$ city models. The absence of 3D building models for larger territories or even whole countries makes some specialized tasks very difficult or even impossible to solve. Thus, there is an evident demand for more 3D building models that experts and researchers could use.

\subsection{DATA}

Different data gathering techniques, including terrestrial and aerial laser scanning or photogrammetry, are used to create 3D building models. Aerial data gathering techniques must be used to create $3 \mathrm{D}$ building models because they enable a rapid and non-selective mapping of large-scale areas. Airborne laser scanning (ALS) and aerial photogrammetry (AP) are conventional methods for a collection of aerial data. The ALS serves to collect information for DSM and especially DTM creation due to the registration of multiple reflections. An ALS laser pulse can pass through vegetation (typically tree crowns) and provide ground height information [4. AP primarily produces seamless orthophoto maps (subsequently: "orthophotos") using vertical images, which capture ground truth from a nadir view. AP is also used to collect oblique aerial photographs to observe the captured scene from multiple viewing angles. Oblique imagery can significantly help with the interpretation of ground features in highly occluded areas. In many countries, orthophotos are acquired on a regular basis to update GIS and other map products. The update frequency usually depends on the size and complexity of the country and may range from one year (e.g., in the Netherlands) [5] to several years (United Kingdom) 6]. Orthophotos and source aerial survey photographs (ASP) represent common and up-to-date type of spatial information 
available for whole countries. Their primary advantage is that they contain not only positional information but also height information when there are sufficient overlaps. They also contain positional and height information, which is uniform in time. Modern image matching algorithms can automatically generate very dense point clouds (PC) from ultra-high resolution stereo images (spatial resolution less than $0.3 \mathrm{~m}$ [7]), which are suitable for the creation of detailed DSMs. Image matching provides height and also colour information for top surfaces but does not penetrate the vegetation like the ALS. 3D building models can be generated successfully with laser-based PCs (LPC) or image-based PCs (IPC). The biggest difference between them is that image matching does not allow the height information to be generated in textureless image parts, such as deep cast shadows and highly reflective materials or in stereoscopic occlusions. Oblique aerial photographs can be used as an alternative or replacement of the vertical imagery to reduce occlusions in cities [8. ALS can fail to sense objects that are highly reflective or which absorb laser beams. These weaknesses have to be taken into account or could be addressed by a fusion of IPC and LPC in highly reliable DSMs [9]. Unfortunately, a simultaneous acquisition of laser and image data from the same flight platform is still not very common.

\subsection{Building outlines}

3D building models could be created purely from spatial data as completely separate objects or their creation could be supported or fully linked to existing $2 \mathrm{D}$ building outlines. Both approaches have advantages and disadvantages in relation to roof structures. The size and shape of building models made solely from aerial data (typically PC) are defined by the size and shape of the roofs. Roofs usually have overhangs over any outer walls, so building outlines derived from roofs could be larger than the real built-up area. Potentially undesirable topological conflicts may also occur when merging these building models with other spatial objects such as road networks and facilities. Therefore, an alternative approach can be employed: using existing building footprints. Building footprints can be obtained from cadastre or national GIS databases that produce large-scale maps and plans. Creating 3D building models from outlines allows them to be combined with other spatial objects from resource databases, significantly increasing their value and usefulness. However, combining existing $2 \mathrm{D}$ data with point clouds may lead to complications. Depending on the type and quality of the resource GIS databases, building shapes may become too general or become shifted. Building outlines from cadastral maps are usually very accurate, but they represent the intersection of the outside walls with the ground 3 and may not necessarily match the shape of a roof that might be simpler or more complex than the building outline.
Any resultant 3D building models might, therefore, not exactly fit to the roofs.

The purpose of 3D building models should be considered when selecting which technique to use. Existing $2 \mathrm{D}$ outlines should be used for the integration into existing databases. For other cases, 3D building models can be generated only from point clouds.

\subsection{D BUILDING MODELS}

Many researchers have examined various aspects of $3 \mathrm{D}$ building reconstruction, with the first studies dedicated to this topic dating back two decades 10 . Our previous publications mentioned some of the early investigations in this area [11, 12. [13] provides a very comprehensive review of methods and principles for an automatic 3D building reconstruction. This review was followed by another work that presented ISPRS benchmark results for building detection and $3 \mathrm{D}$ building reconstruction [14, 15]. The following text is structured into sections according to the type of spatial data.

\subsubsection{LASER-BASED POINT CLOUDS}

Many studies deal with the creation of 3D building models exclusively using LPC [5, 16, 17]. Algorithms based on roof topology graphs [5, 17, 18, represent well-developed approaches using high-density PC (20 points $/ \mathrm{m}^{2}$ ). Global optimization solutions to create roof models from low-density LPC (at least 3 points $/ \mathrm{m}^{2}$ ) have also been introduced [16. Such solutions require $\mathrm{PC}$ segmentation into roof planes, the extraction and regularization of building boundaries and step edges, partitioning building bounding boxes into volumetric cells and categorizing them as inside or outside based on a visibility analysis [16. The faces between the inside and outside cells form the reconstructed 3D building model. Authors [16] stated that their solution was robust in terms of missing points due to occlusions, but their results were fully dependent on the completeness of input roof planes. Regardless of the LPC approach chosen, it is important to realize that building boundaries (outlines) are derived from points classified as roofs and thus could be larger than the real built-up area.

\subsubsection{IMAGE-BASED POINT CLOUDS}

A recently published study introduced a generation of LoD2 building models from photogrammetric point clouds without using any ancillary data such as building footprints [19]. In this work, the building generation was based on PC segmentation using a regiongrowing algorithm, extraction primitives using Random Sample Consensus [20, and creation of 3D building models with PolyFit software. Polygonal surface reconstruction from point clouds (PolyFit) is a framework for the generation of simple polygonal surface models from intersecting planes 21. According to the software authors [21], PolyFit handles noise, outliers and missing data in PC. 


\subsubsection{Point ClOUdS AND IMAGE DATA}

Technically very fascinating papers have described the connection of height and image data to create building models $[22,23$. Image data can be successfully used to detect roof edges. Detected roof edges can form the final vertices of a reconstructed roof model. A solution presented by [22] was based on the extraction of roof vertices from true-orthophotos $(0.1 \mathrm{~m} / \mathrm{pixel})$ using Canny edge detector and their integration with DSM $(0.1 \mathrm{~m} / \mathrm{pixel})$ created from IPC to form closed-cycles of roof planes. Detected roof edges can also support an extraction of roof planes from PC 23. Methods based on the extraction of roof planes using a region-growing algorithm from high-density LPC (35 points $\left./ \mathrm{m}^{2}\right)$ supported by edges extracted from UHR orthoimages $(0.05 \mathrm{~m} /$ pixel) have been described in detail in 23 .

\subsubsection{LASER-BASED POINT CLOUDS AND OUTLINES} Fusing the use of building outlines from a national topographic database with planimetry accuracy of 1-2 $\mathrm{m}$ and laser scanning elevation data to produce a nationwide 3D landscape model was presented in the Netherlands [5]. Point clouds with reduced density from 20 to 3 points $/ \mathrm{m}^{2}$ were used to create a 3D landscape model of all topographic objects in LoD0 and building models in LoD1 with flat, horizontal roofs. In this study, fusing the very accurate point clouds with less accurate outlines might complicate the creation of resultant building models. This was the reason for choosing low-quality LoD1 instead of LoD2 building models.

\subsubsection{IMAGE-BASED POINT CLOUdS AND OUTLINES} Three European national mapping agencies (United Kingdom, Ireland and Spain) tested oblique aerial datasets to generate very dense point clouds, textured polygonal meshes and 3D building models in LoD2 from known footprints [8]. City Modeller, module of the Tridicon/Hexagon software, was used for creation of $3 \mathrm{D}$ building models. According to the authors, point clouds produced from oblique imagery are cleaner in comparison with the conventional nadir images and additionally contain points on building facades. However, medium format oblique cameras and image overlaps up to $80 \%$ lead to more flight hours and thus to higher aerial survey costs. Furthermore, a large number of images affects processing time and storage requirements.

\subsubsection{OUTLINES AND ATTRIBUTES}

Fast automatic generation of 3D building models from outlines linked to attributes data (the number of stories and the type of roof) is also possible [24]. This approach requires reshaping the building polygons into orthogonal form and partitioning them into rectangles. For each rectangle generates a basic 3D building model according to linked attributes. An extension of this approach for automatic generation of buildings with generally-shaped roof models is possible using non-orthogonal footprints and straight skeleton computation [25]. Unfortunately, the straight skeleton technique can only produce hipped roof models. Complete elimination of $\mathrm{PC}$ analysis greatly simplifies the problem creating LoD2 building models but reduces the model's exactness.

The rest of this paper is organized as follows. Our aims and motivation are presented in Section 2 Section 3 contains a detailed description of the technique we developed, supported by many sample images. The evaluation of results and discussion are provided in Section 4 Section 5 summarizes conclusions and possible future plans.

\section{AIMS}

This work builds on our previous publications [11, 12 ] that were focused on the comparison of existing commercial software solutions, using PC (ENVI LiDAR) and also existing outlines (INPHO Building Generator), to create $3 \mathrm{D}$ building models. The building models created in our previous investigations were not satisfactory. INPHO Building Generator building models were simple and topologically correct, but a large number of them did not correspond to real forms. ENVI LiDAR could create realistic and even complex 3D building models but were composed of many overlapping polyhedrons and thus contained many topological errors due to a missing topology control. Because of these imperfections, we decided to develop our own method for an automatic creation of topologically correct building roof models. Only such roof models can be used to produce useful 3D building models corresponding to reality. Our method uses vector building outlines together with image and height information derived from ASP to reconstruct roof models. Our approach incorporates current requirements of the local national mapping agency (NMA) of the Czech Republic, including using existing building outlines (positional accuracy up to 2 meters, usually up to $1 \mathrm{~m}$ ) and currently available ultra-high resolution ASP (ground sample distance 0.15-0.25 m) for the creation of $3 \mathrm{D}$ building models without the need to collect extra spatial data.

\section{Description OF THE ROOF MODEL CONSTRUCTION}

The technique developed combines image and height information in raster format derived from standard top-view ASP with approximate $55 \%$ forward and $20 \%$ side overlaps. Image data represents colour orthophotos (resolution $0.25 \mathrm{~m} / \mathrm{pixel}$ ) from the Czech NMA (Land Survey Office). Height data represents normalized DSM (nDSM) created by subtracting DTM from DSM. All models are in a raster form with a resolution of $1 \mathrm{~m} /$ pixel. The DTM used comes from Land Survey Office data obtained by ALS. The DSM used comes from Forest Management Institute datasets and was generated by a fusion of stereo-image 


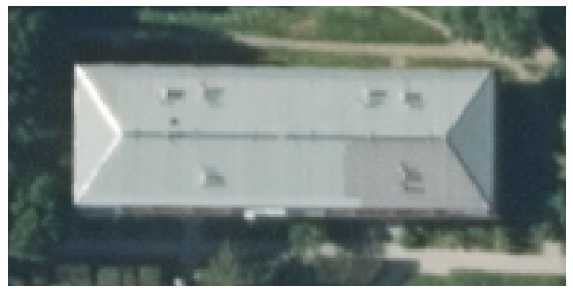

Figure 1. Orthophoto.

PCs into the final model and projection of the final model to a plane. The PCs were generated with the enhanced Automatic Terrain Extraction (eATE) module to ERDAS IMAGINE or IMAGINE Photogrammetry (formerly Leica Photogrammetry Suite). The eATE is a dense image matching algorithm. It uses a pixel by pixel correlation technique to generate very dense point clouds from a stereo imagery coverage [26]. In our case, the normalized cross-correlation was used to produce point clouds with a density of 1 point $/ \mathrm{m}^{2}$. The generated PC has a lower quality (height deviations up to $1 \mathrm{~m}$ ) than the results obtained by stateof-the-art image matching algorithms [27, 28] but it was fully sufficient for our needs. Using a DTM generated from an ALS dataset for the normalization of DSM, we introduced another height error into nDSM. In addition, we converted the final nDSM to 8-bit (integers/meters from 0 to 255 range) for optimization reasons. Height errors could theoretically be up to 2 meters using the processing steps we employed. However, this was not a complication because our approach did not require high-quality nDSM used. An example of an orthophoto used in our study is shown in Figure 1. A small north-west shift in the building roof image in relation to the ground is recognizable (Fig. 1). This is a radial image shift of above-terrain objects due to the central projection. The orthophoto used was not a true orthophoto because of small overlaps between images.

Building footprints were obtained from the Czech NMA, Land Survey Office, which administers the national GIS database (Fundamental Base of Geographic Data of the Czech Republic) 29]. Building outlines were processed one by one. Three approximate roof height values (top, bottom, overall height) based on nDSM were calculated for each building footprint. Top height was calculated to be the 95 th percentile of all nDSM values in the building footprint. Roof bottom height was calculated as the third quartile of all nDSM values within a one-meter distance around (inside and outside) the building outline. Roof overall height was calculated as top height minus roof bottom height.

The color orthophoto was converted to grayscale values (luma) as a weighted sum of the red $(R)$, green $(\mathrm{G})$, blue (B) components with the formula $R \cdot 0.299+G \cdot 0.587+B \cdot 0.114$. Roof edges were detected from grayscale orthophoto with a Line Segment Detector (LSD). The LSD algorithm detects

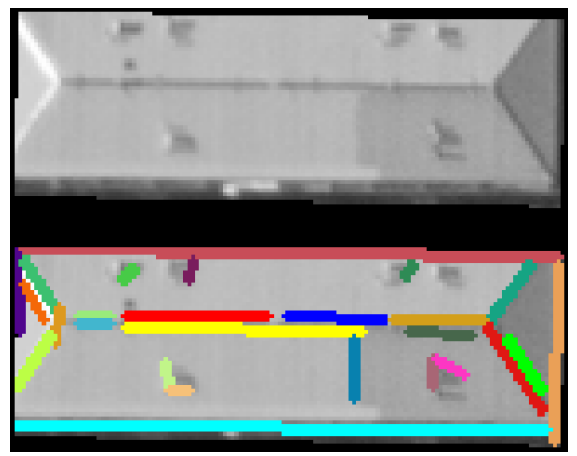

Figure 2. Orthophoto of building with hip roof [top] and edges detected with the Line Segment Detector [bottom].

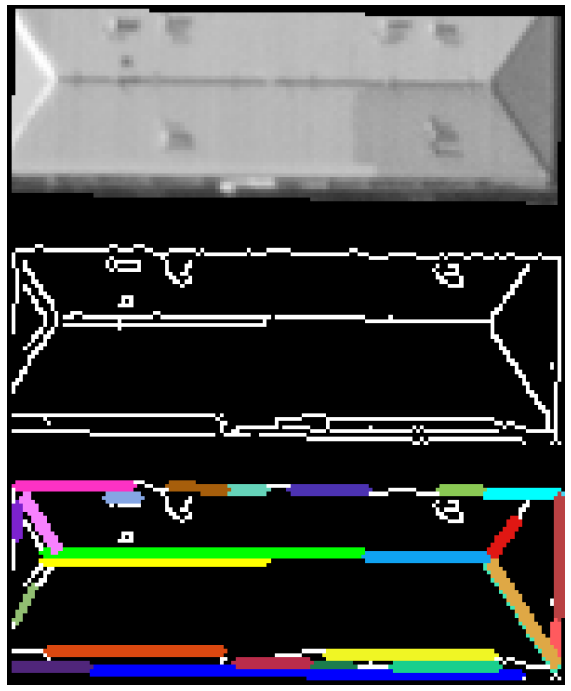

FiguRE 3. Orthophoto of building with hip roof [top] and the result of Canny edge detector with automatic parameter tuning [middle] followed by a probabilistic Hough transform [bottom].

locally straight contours (also called line segments) on grey-level images without a parameter tuning [30]. According to 31, LSD is an automatic image analysis tool working in a manner similar to a human perception because the level of detail depends on the size of the entire image being analysed. We selected this edge detection algorithm because it achieves satisfactory results without any need for parameter adjusting, is very fast, and is a part of the Open Source Computer Vision Library (OpenCV) [32. It is thus the ideal edge detection algorithm for fully automated procedures. Its disadvantage is that it works only with grey-level images, so some edges can be lost during the colour to grayscale conversion. Nonetheless, this is not a disadvantage for the detection of roof edges, typically defined by roof planes having different brightness levels depending on their exposure to the sun. The performance of the LSD was compared to the standard edge detection technique based on the Canny edge detector 33 with an automatic parameter tuning 34] followed by probabilistic Hough transform [35. Results of both edge detection ap- 


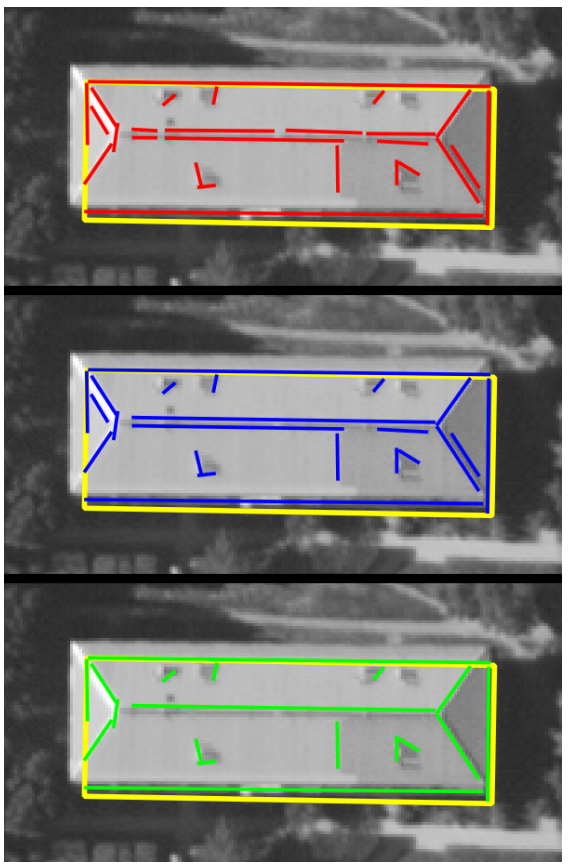

Figure 4. Orthophoto of building with hip roof and building outline (solid line), edges detected [top], edges merged [middle] and edges filtered [bottom].

proaches for the same image are in Figures 2 and 3 Both approaches detected edges that represent main roof edges and other elements, such as dormers and building outlines. Identified edges on building outlines were detected due to the sudden change of pixel values on the boundaries of the clipped orthophoto. According to a visual inspection, LSD (Fig. 2 provided better results. The edges detected matched each other and did not overlap, which was important for a further post-processing. The post-processing consists in merging adjacent edges and their filtration to remove duplicates.

The proposed method employed in our study was based on the roof edge detection and categorization. However, edges detected with any method must represent meaningful roof elements (ridges, hips, valleys, dormers) for a proper categorization. Unfortunately, some roof elements were fragmented (ridge in Fig. 2) or duplicated (hips in Fig. 22). That is why we merged detected edges and removed duplications prior to the categorization. The edges detected were merged based on the following three conditions: similar angle $\left(\right.$ angle $_{\text {diff }} \leq 9^{\circ}$ ), adjacency (endpoints distance $\leq 1.25 \mathrm{~m}$ ), and the new edge formed by joining them must be longer than each of them. Subsequently, shorter parallel edges (within $1 \mathrm{~m}$ ) were filtered out. The results of merging and filtration are shown in Figure 4. Here, edges representing the ridge have been correctly merged and shorter duplicate edges have been removed.

Having executed the previous steps, it was possible to perform an initial edge categorization based on the analysis of height information from nDSM and the

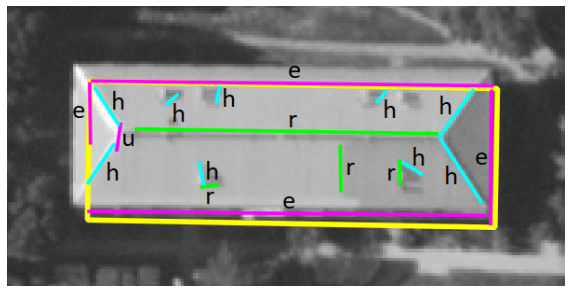

Figure 5. Categorized edges: ridges (r), hips/valleys (h), eaves (e), uncategorized (u).

spatial relationships between edges detected and the building footprint. This unique approach was based on the detection of ridges, hips/valleys, and eaves.

The roof ridge was recognized using several characteristic features. The main specific features included parallelism with at least one building outline $\left(\right.$ angle $_{\text {diff }}$ $\leq 9^{\circ}$ ), ideally no (or a very small) difference in the height of endpoints (height diff $\leq 2 \mathrm{~m}$ ), and - for the main ridge - the height of endpoints should correspond to the primary top height (height $\mathrm{diff} \leq 2 \mathrm{~m}$ ). However, hips and valleys were categorized using different criteria. The edge was marked as a hip/valley if it was non-parallel $\left(\right.$ angle $_{\text {diff }}>9^{\circ}$ ) and close (distance $>1 \mathrm{~m}$ ) to the building outline. For edges $2.5 \mathrm{~m}$ and longer, the height difference of the endpoints had to be at least $0.5 \mathrm{~m}$. The height difference of endpoints was not checked for edges shorter than $2.5 \mathrm{~m}$; thus, only the first two conditions were sufficient. The remaining edges were classified as eaves/gutters or uncategorized edges. Eaves or gutters were parallel (angle diff $\leq 9^{\circ}$ ), close (distance $<1 \mathrm{~m}$ ) to the building outline, and ideally had no (or a very small) difference in the height of endpoints (height diff $\leq 2 \mathrm{~m}$ ) and corresponded to the primary roof bottom height (height ${ }_{\text {diff }} \leq 2 \mathrm{~m}$ ). All other edges that did not meet the relevant criteria for specific roof elements were classified as uncategorized. Figure 5 shows the results of the categorization. All edges representing the main roof elements were properly categorized.

Ridges and hips/valleys were additionally sorted. Ridges were sorted according to height (highest to lowest) and the hips/valleys by length (longest to shortest). The roof model reconstruction started with the highest ridge and its adjacent hips/valleys. Hips/valleys were considered adjacent to a ridge if one of their endpoints was close (distance $<1.5 \mathrm{~m}$ ) to one of the ridge endpoints. Hips and valleys were further differentiated from each other according to their orientation to the ridge. Hips formed an obtuse angle with the ridge while valleys formed an acute angle with the ridge.

A multi-plane roof type (hip/half-hip, saddle, dormer, or pyramid) was determined according to the existence and position of the ridge in relation to the building outline and adjacent hips and valleys. A hip (and half-hip) roof was defined as a single ridge roof with at least one adjacent hip (Fig. 5p. A saddle roof was defined as a single ridge roof with at least one 


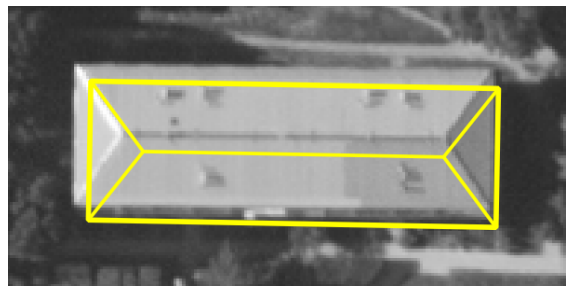

FiguRE 6. Reconstructed roof model.

endpoint (within distance $<1 \mathrm{~m}$ ) near to the building outline and having no adjacent hips. A dormer was defined as a single ridge roof with at least one adjacent valley and no hips. A pyramid roof had no ridge but at least one hip. A mono-plane roof type (flat or shed) was determined based on a height analysis of eaves (outline segments) and approximate roof height values. A flat roof had similar heights for outline segments, top and bottom. A shed roof had equally tilt parallel eaves. A roof was classified as unknown if the edge configuration or height analysis did not match the previous roof type definitions.

Edges detected (ridges, hips, valleys) by our method could have positional offset from their real position due to a conventional orthophoto in which the image of the roof elements might have radially shifted. Thus, the position of edges detected could not be used directly for the creation of roof models as in [22. Thus, our method used information about angles, lengths, and the topology of edges detected for the creation of models according to additional rules (or rather, constraints) for individual roof types. Our method created roof models only for building outlines in the shape of a polygon with two parallel sides (subsequently: "polygon"). There was one common constraint for all roof types with a ridge. The ridge was always constructed exactly in the middle of the polygon parallel sides and its angle was calculated as the average of angles of the polygon parallel sides if they had angles similar to the detected ridge (angle diff $\leq 9^{\circ}$ ). In the following text, this constraint referred to as "the rules".

Creation of a hip roof began with the construction of a ridge according to the rules. The length of the constructed ridge corresponded to the length of the detected ridge. Any ridge was constructed from information about the angle and length from the centroid (for a rectangle polygon) or a fixed ridge endpoint (for a side roof polygon). Hips, line segments that connect the endpoints of the constructed ridge and the nearest vertices of the polygon, were then created and our algorithm checked the angles of the constructed and detected hips. If at least one angle of the constructed hip corresponded to one angle of the detected hip, the roof type was confirmed. The vector model (skeleton) was created by joining the polygon, the ridge, and the hips. Figure 6 shows the reconstructed hip roof model. However, if the angles of the constructed and detected hips did not match, the algorithm continued by creating a half-hip roof.

The half-hip roof model creation was similar to that of the hip roof. The ridge design was identical. The main difference was in the construction of the hips. The hips were created based on the angles detected. From the hips detected, those which formed a similar angle to a ridge $\left(\right.$ angle $\left._{\text {diff }} \leq 9^{\circ}\right)$ were selected and their average angle was calculated. If only one hip was detected, its angle value was used. The hips were rendered as line segments connecting the endpoints of the ridge and the intersections of the hip half-lines and the perpendicular sides of the polygon. Unfortunately, there was no control in the rendering of the half-hip roof because all the available values had already been used to construct the roof model. A skeleton of the half-hip roof was created by joining the polygon, the ridge, and the hips.

The gable roof model was composed only of the ridge. As in previous cases, the ridge was designed according to the rules and rendered only using the information about the angle from the centroid or the fixed ridge endpoint. The algorithm checked the length of the constructed ridge against the length of the detected ridge. In case that the difference was smaller than the defined threshold $\left(\right.$ length $_{\text {diff }}<2 \mathrm{~m}$ ), a gable roof model was validated.

A pyramid roof skeleton consisted of 4 hips connecting the centroid and the vertices of the rectangle. It was not appropriate to compare the lengths of the hips detected against those rendered because the opposite hips could be joined during the edge merging process to the diagonal. The algorithm checked the angles of both detected and rendered hips. If an angle of at least one rendered hip corresponded to an angle of one detected hip, a pyramid roof type was confirmed.

If the building outline shape was complex, the building was decomposed. Building decomposition consisted of dividing the outline into individual line segments and their extensions inward to form inner line segments (intersections). The intersections could cross each other to form smaller inner line segments. Our aim was to combine outline segments and inner line segments (further line segments) to form nearest parallel line segments on both sides of the ridge. The line segments found defined two parallel sides of a polygon. Depending on the existence of valleys, a polygon had to meet certain criteria. If there were no valleys adjacent to the ridge, the shape of a polygon should have corresponded to a rectangle. For a saddle roof, the ridge and parallel rectangle sides were approximately the same length $\left(\right.$ length $\left._{\text {diff }}<2 \mathrm{~m}\right)$. For a hip/half-hip roof, parallel rectangle sides were about the same length or longer than the ridge. If there were valleys adjacent to the ridge, the shape of the polygon was complex and dependent on the number of valleys. Such a polygon usually represented a side roof rectangle and contained a so-called fixed ridge endpoint. A fixed ridge endpoint was defined as the 


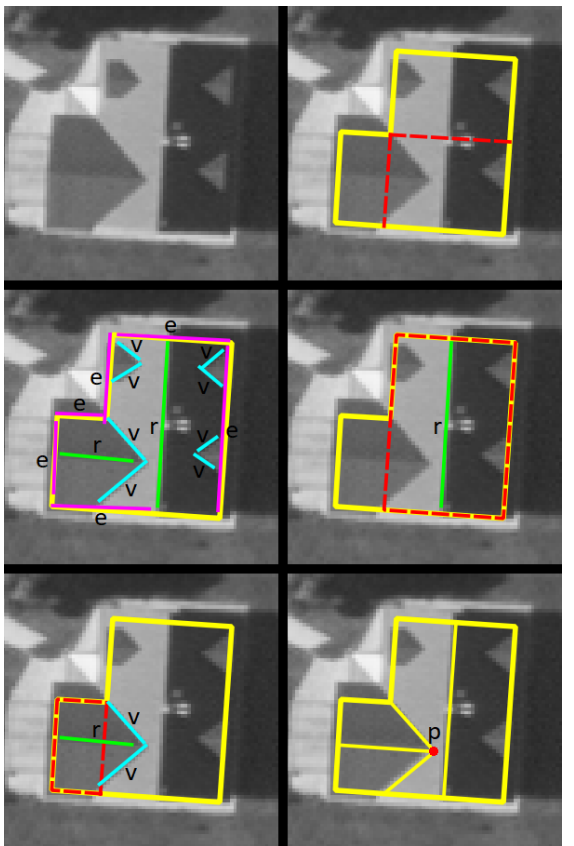

FiguRE 7. Orthophoto of complex building with gable roof [top left], building outline (solid line) and intersections (dashed line) [top right], categorized edges (legend as in Fig. 5 [middle left], main ridge (r) and polygon (dashed line) [middle right], side ridge (r), valleys (v) and polygon (dashed line) [bottom left], reconstructed roof model with a fixed ridge endpoint (p) [bottom right].

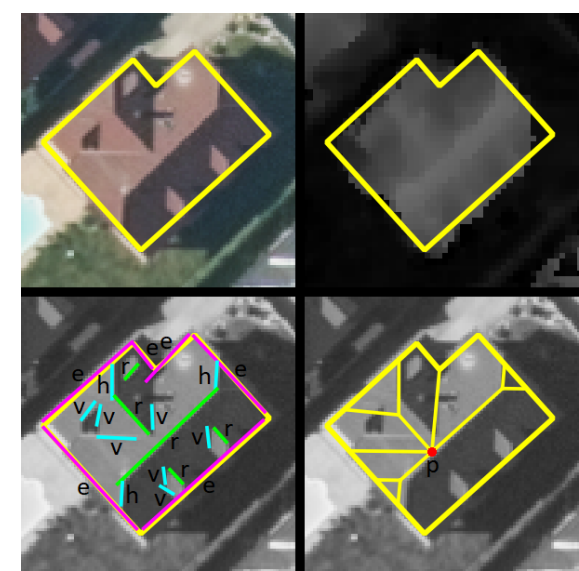

FIGURE 8. Orthophoto of complex building with halfhip roof and building outline (solid line) [top left], nDSM with $0.5 \mathrm{~m} /$ pixel resolution and building outline [top right], categorized edges (legend as in Fig. 5) [bottom left], and reconstructed roof model with a fixed ridge endpoint (p) [bottom right].

intersection of a valley half-line and a ridge line following the rules. An example of processing a complex building outline is shown in Figure 7

\section{Results AND Discussion}

The strengths of our method include its robustness and topological correctness defined by rules for the reconstruction of individual roof types. Figure 8 shows

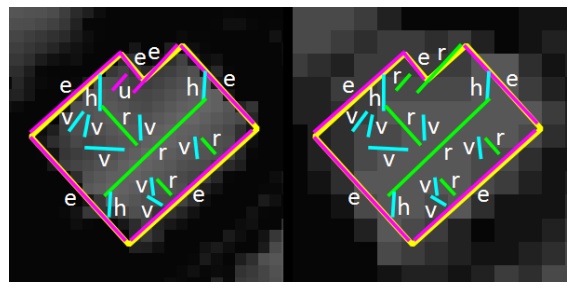

Figure 9. nDSM with resolution $1 \mathrm{~m} /$ pixel and categorized edges (legend as in Fig. 5p [left], nDSM with resolution $2 \mathrm{~m} /$ pixel and categorized edges [right].

an example of a roof model created for one L-shaped building with a half-hip roof. Not all half-hips were detected and valleys detected were shorter than in reality. Despite the incompleteness of the detection, a satisfactory roof model was reconstructed, demonstrating the robustness of our approach. Unfortunately, the side ridge was incorrectly connected directly to the main ridge, but the algorithm prevented the mutual crossing of the main and side ridges that would occur due to the spacing and angle of detected valleys. The roof model created was thus topologically correct. In this example (Fig. 8), nDSM with $0.5 \mathrm{~m} /$ pixel resolution created from IPC was used.

Another significant advantage of our solution lies in the possibility of using nDSMs with different levels of quality. As noted above, the roof edges were detected using orthophotos and nDSM only helped with their categorization. Thus, the quality of orthophotos employed using such a method is important but the quality of a nDSM is less important. Figure 9 illustrates the results of the edge categorization using nDSMs with different resolutions. Using lower quality nDSM led to several changes in the categorization of edges at the top of the building. However, these changes did not affect the construction of the roof model because the categorization of key edges remained unchanged. This demonstrates that nDSMs with quality variances and origins can be used to reconstruct roof models without any significant side effects.

We tested our method on a small dataset containing approximately 30 buildings of various shapes (number of vertices: 4-16) and roof complexity (number of roof types: 1-3). Excellent results were achieved for simple and medium complex buildings. Examples of the evaluation are shown in Figure 10. Stereo photogrammetric measurements were used to obtain the reference data in the form of corresponding $3 \mathrm{D}$ points (colour dots in Fig. 10). The root mean square error (RMSE) [36] was calculated separately between the vertices of the building outline (input data) and roof skeleton (extracted data) and the reference points for every building. The average RMSE was $0.73 \mathrm{~m}$ for the building outline and $0.92 \mathrm{~m}$ for the roof skeleton. Unfortunately, the results for very complex cases were unsatisfactory. The model reconstruction failed due to missing key roof edges. This situation occurred when a roof was inappropriately illuminated, so some edges 


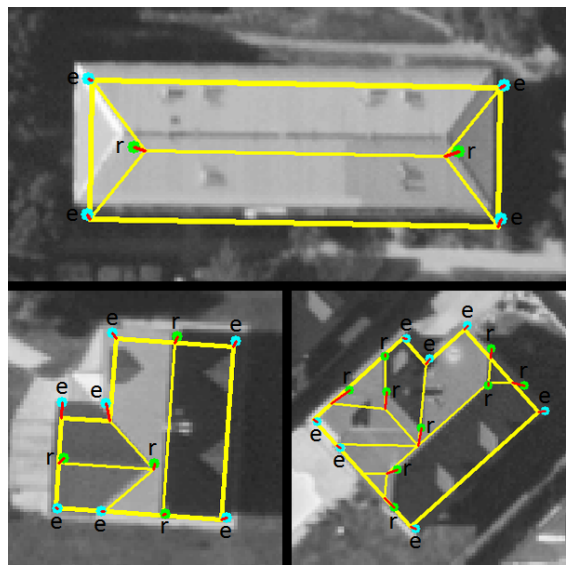

Figure 10. Examples of evaluation with reference vertices as eaves (e), ridges/half-hips (r) and shifts between corresponding vertices (short line).

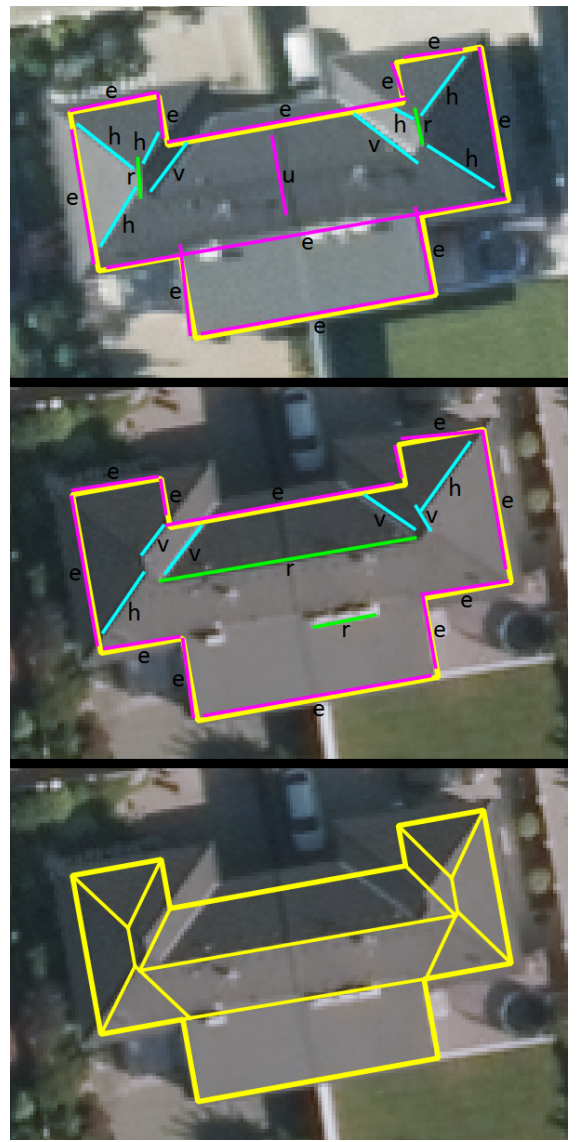

FiguRE 11. Orthophoto of complex building with mixed roof type and categorized edges (legend as in Fig. 5) from epoch 1 (2014) [top], epoch 2 (2016) [middle] and reconstructed roof model [bottom].

could not be properly detected. For such situations, we propose using multitemporal images because these can lead to significant improvements by allowing more roof edges to be detected. Figure 11 shows colour orthophotos of a complex building with mixed roof type in two epochs (2014 and 2016). Different roof edges were detected in each epoch and their combination enabled a reconstruction of the roof model.
Weaknesses of our method include the limitations resulting from the application of rules for individual roof types. For example, the current implementation does not permit reconstruction of roof ridges except in the middle or on the outline of buildings. Also, undefined roof types, such as sawtooth, mansard, butterfly, and dome cannot be created. Another drawback is the way in which the heights of detected edges are determined. The radial shift of a building roof image is not taken into account. Thus, the roof edges detected are slightly horizontally shifted. However, for their categorization, we used the endpoint heights determined from the positionally correct nDSM raster. An inaccurate determination of the heights could, in extreme cases, lead to erroneous edge categorizations and, therefore, make it impossible to reconstruct a roof model. For this reason, we recommend using only the central part of the orthophotos, where the image shift is minimal or very small. This is the standard procedure for creating a seamless orthophoto mosaic used in our case. It is clear that using a trueorthophoto is ideal for the edge detection, oblique images are extremely inappropriate. However, oblique images have many benefits for the creation of highquality DSMs using dense image matching. In order to create accurate $3 \mathrm{D}$ building models, it is necessary to re-determine all elevations from the $\mathrm{nDSM}$ (or DSM) after the roof reconstruction.

\section{Conclusions}

In this paper, we introduced an approach for an automatic reconstruction of roof models using planar $/ 2 \mathrm{D}$ building footprints, a nadir orthophoto, and nDSM. All data types used were typical spatial datasets often managed by national mapping agencies. The solution presented was developed and tested using real standard resolution production data $(0.25 \mathrm{~m} /$ pixel for orthophotos and $1 \mathrm{~m} /$ pixel for DSM) and illustrated how it is not necessary to acquire extremely high resolution spatial datasets. Because of commonly available datasets were employed, the method described here is widely applicable and inexpensive. The roof model reconstruction was based on the extraction of $2 \mathrm{D}$ roof edges from an orthophoto and categorized using height and spatial relationship information. Categorized edges were used for determining the type of the roof and its key parameters (especially hip angles). Buildings with a complex footprint were solved in part through a decomposition into simple shapes according to ridges detected and adjacent valleys. The simpleto-use method described here allows for the creation of visually attractive building roof models composed of gable, hip, half-hip, tent, flat, and shed roof types. Simple parameter tuning consisted of defining several variables with angle and length thresholds. The preliminary results presented in this paper are very promising. Future work will focus on the possibility of using the fully blocked building footprints and step edges extraction with more accurate nDSMs and the 
implementation of other roof types. In addition, future plans include the creation of CityGML standard $3 \mathrm{D}$ building models and a real-world implementation in cooperation with the Land Survey Office in the Czech Republic.

\section{ACKNOWLEDGEMENTS}

This work was supported by the Czech Technical University in Prague Student Grant Competition (grant number SGS19/048/OHK1/1T/11).

\section{REFERENCES}

[1] M. Hůlková, K. Pavelka, E. Matoušková. Automatic classification of point clouds for highway documentation. Acta Polytechnica 58:165-170, 2018. DOI:10.14311/AP.2018.58.0165.

[2] G. Gröger, L. Plümer. CityGML - Interoperable semantic 3D city models. ISPRS Journal of Photogrammetry and Remote Sensing 71:12-33, 2012. DOI:10.1016/j.isprsjprs.2012.04.004

[3] M. Med, P. Souček. Analysis and implementation of application schemas for the INSPIRE buildings theme. Acta Polytechnica 56:291-300, 2016. DOI:10.14311/ap.2016.56.0291

[4] G. Vosselman, H.-G. Maas (eds.). Airborne and Terrestrial Laser Scanning. Dunbeath: Whittles. CRC Press, 2010.

[5] G. Vosselman, S. O. Elberink, M. Post, et al. From nationwide point clouds to nationwide 3D landscape models. Photogrammetric Week pp. 247-256, 2015.

[6] C. S. Gladstone, A. Gardiner, D. Holland. A semi-automatic method for detecting changes to Ordnance Survey topographic data in rural environments. In GEOBIA, pp. 396-401. 2012.

[7] R. Qin, J. Tian, P. Reinartz. 3D change detection Approaches and applications. ISPRS Journal of Photogrammetry and Remote Sensing 122:41-56, 2016. DOI:10.1016/j.isprsjprs.2016.09.013

[8] F. Remondino, I. Toschi, M. Gerke, et al. Oblique aerial imagery for NMA: some best practices. In L. Halounova (ed.), Proceedings of the XXIII ISPRS Congress - From human history to the future with spatial information, vol. III-2, pp. 639-645. International Society for Photogrammetry and Remote Sensing (ISPRS), 2016. DOI:10.5194/isprs-archives-XLI-B4-639-2016.

[9] G. Mandlburger, K. Wenzel, A. Spitzer, et al. Improved topographic models via concurrent airborne lidar and dense image matching. ISPRS Annals of Photogrammetry, Remote Sensing and Spatial Information Sciences IV-2/W4:259-266, 2017. DOI:10.5194/isprs-annals-IV-2-W4-259-2017.

[10] N. Haala, C. Brenner. Extraction of buildings and trees in urban environments. ISPRS Journal of Photogrammetry and Remote Sensing 54:130-137, 1999. DOI:10.1016/S0924-2716(99)00010-6

[11] V. Hron, V. Kostin, L. Halounová. Comparison of software solutions for automatic generation of $3 \mathrm{D}$ building models. In International Multidisciplinary Scientific GeoConference Surveying Geology and Mining Ecology Management, vol. 1, pp. 513-520. 2014. DOI:10.5593/SGEM2014/B21/S8.065.
[12] V. Hron, L. Halounová. Automatic generation of 3D building models from point clouds. In I. Ivan, I. Benenson, B. Jiang, et al. (eds.), Geoinformatics for Intelligent Transportation, pp. 109-119. 2014. DOI:10.1007/978-3-319-11463-7_8.

[13] N. Haala, M. Kada. An update on automatic 3D building reconstruction. ISPRS Journal of Photogrammetry and Remote Sensing 65:570-580, 2010. DOI:10.1016/j.isprsjprs.2010.09.006

[14] F. Rottensteiner, G. Sohn, J. Jung, et al. The ISPRS benchmark on urban object classification and 3D building reconstruction. ISPRS Annals of Photogrammetry, Remote Sensing and Spatial Information Sciences I3:293-298, 2012. DOI:10.5194/isprsannals-I-3-293-2012.

[15] F. Rottensteiner, G. Sohn, M. Gerke, et al. Results of the ISPRS benchmark on urban object detection and $3 \mathrm{D}$ building reconstruction. ISPRS Journal of Photogrammetry and Remote Sensing 93:256-271, 2014. DOI:10.1016/j.isprsjprs.2013.10.004

[16] J. Yan, W. Jiang, J. Shan. A global solution to topological reconstruction of building roof models from airborne lidar point clouds. ISPRS Annals of Photogrammetry, Remote Sensing and Spatial Information Sciences III-3:379-386, 2016. DOI:10.5194/isprs-annals-III-3-379-2016

[17] B. Xiong, S. Oude Elberink, G. Vosselman. A graph edit dictionary for correcting errors in roof topology graphs reconstructed from point clouds. ISPRS Journal of Photogrammetry and Remote Sensing 93:227-242, 2014. DOI:10.1016/j.isprsjprs.2014.01.007.

[18] B. Xiong, M. Jancosek, S. O. Elberink, G. Vosselman. Flexible building primitives for 3D building modeling. ISPRS Journal of Photogrammetry and Remote Sensing 101:275-290, 2015. DOI:10.1016/j.isprsjprs.2015.01.002.

[19] I. Pârvu, F. Remondino, E. Özdemir. LOD2 building generation experiences and comparisons. Journal of Applied Engineering Sciences 8:59-64, 2018. DOI:10.2478/jaes-2018-0019

[20] M. A. Fischler, R. C. Bolles. Readings in computer vision: Issues, problems, principles, and paradigms. chap. Random Sample Consensus: A Paradigm for Model Fitting with Applications to Image Analysis and Automated Cartography, pp. 726-740. Morgan Kaufmann Publishers Inc., San Francisco, CA, USA, 1987. DOI:10.1016/b978-0-08-051581-6.50070-2.

[21] L. Nan, P. Wonka. PolyFit: Polygonal surface reconstruction from point clouds. In IEEE International Conference on Computer Vision, pp. 2372-2380. 2017. DOI:10.1109/iccv.2017.258.

[22] A. McClune, J. Mills, P. Miller, D. Holland. Automatic 3D building reconstruction from a dense image matching dataset. ISPRS International Archives of the Photogrammetry, Remote Sensing and Spatial Information Sciences XLI-B3:641-648, 2016. DOI:10.5194/isprsarchives-XLI-B3-641-2016.

[23] M. Awrangjeb, C. Zhang, C. S. Fraser. Automatic extraction of building roofs using LIDAR data and multispectral imagery. ISPRS Journal of Photogrammetry and Remote Sensing 83:1-18, 2013. DOI:10.1016/j.isprsjprs.2013.05.006 
[24] K. Sugihara, T. Murase, X. Zhou. Automatic generation of $3 \mathrm{D}$ building models from building polygons on digital maps. In Proceedings of the International Conference on 3D Imaging. 2015. DOI:10.1109/ic3d.2015.7391817.

[25] T. Murase, K. Sugihara. Automatic generation of 3D building models for environmental education by straight skeleton computation. In International Conference on Signal Processing, pp. 1040-1045. 2018. DOI:10.1109/icsp.2018.8652493

[26] Hexagon Geospatial. IMAGINE Photogrammetry Brochure. https://www .hexagongeospatial.com/ brochure-pages/imagine-photogrammetry-brochure, 2018. Accessed: 1 May 2019.

[27] F. Remondino, M. Spera, E. Nocerino, et al. State of the art in high density image matching. The Photogrammetric Record 29:144-166, 2014. DOI:10.1111/phor.12063

[28] N. Haala. Dense image matching final report. Tech. rep., European Spatial Data Research, 2014. Official Publication No 64.

[29] Geoportál ČÚZK. ZABAGED®- planimetric components - introduction. https://geoportal.cuzk. cz/Default . aspx?lng=EN\&mode=TextMeta\&text= dSady_zabaged\&side=zabaged\&menu=24, 2019. Accessed: 20 May 2019.

[30] R. Grompone von Gioi, J. Jakubowicz, J.-M. Morel, G. Randall. LSD: A fast line segment detector with a false detection control. IEEE Transactions on Pattern Analysis and Machine Intelligence 32:722-732, 2010. DOI:10.1109/TPAMI.2008.300
[31] R. Grompone von Gioi, J. Jakubowicz, J.-M. Morel, G. Randall. LSD: A line segment detector. Image Processing On Line 2:35-55, 2012. DOI:10.5201/ipol.2012.gjmr-lsd

[32] OpenCV 3.0.0-dev documentation. Feature Detection. https://docs.opencv.org/3.0-beta/ modules/imgproc/doc/feature_detection.html, 2014. Accessed: 1 May 2019.

[33] J. Canny. A computational approach to edge detection. IEEE Transactions on Pattern Analysis and Machine Intelligence 8:679-698, 1986. DOI:10.1109/TPAMI.1986.4767851

[34] A. Rosebrock. Zero-parameter, automatic Canny edge detection with Python and OpenCV. https://www.pyimagesearch.com/2015/04/06/ zero-parameter-automatic-canny-edgedetection-with-python-and-opencv/ 2015. Accessed: 1 May 2019.

[35] J. Matas, C. Galambos, J. Kittler. Robust detection of lines using the progressive probabilistic hough transform. Computer Vision and Image Understanding 78:119-137, 2000. DOI:10.1006/cviu.1999.0831

[36] J. Avbelj, R. Müller. Quality assessment of building extraction from remote sensing imagery. In 2014 IEEE Geoscience and Remote Sensing Symposium, pp. 3184-3187. 2014. DOI:10.1109/IGARSS.2014.6947154. 